Pacific Journal of Mathematic 


\section{EUCLIDEAN AND WEAK UNIFORMITIES}

\section{J. R. ISBELL}

Introduction. This paper is a study in the structure of some special classes of uniform spaces. In outline, machinery is developed in successive stages, roughly two stages. The first stage is illustrated by an unsuccessful attack on the characterization of subspaces of Euclidean spaces, in the usual uniform structure. The second stage leads to a characterization of those uniform spaces which are subspaces of Euclidean spaces in the finest structure consistent with the topology.

The main tool in the second stage is a covariant functor on uniform spaces to uniform spaces which is closely analogous to the derivative, the main tool employed by Ginsburg and the author in [4]. It yields also a number of results which complement, and a couple which improve, results of [4] and of [5].

That tool is inapplicable to the study of the usual Euclidean uniform structure. The approach attempted is to get a subspace of $E^{n}$ as the inverse limit of the nerves of its uniform covering, or of any basis of uniform coverings. Indeed there is a basis of coverings whose nerves are uniformly equivalent to subspaces of $E^{n}$-Euclidean coverings, let us say, and the nerves, Euclidean complexes-and in some sense one can set up an inverse system of mappings on these nerves " uniformly" within $E^{n}$. The contribution of this paper is to formalize this approach and clear away imaginary difficulties, leaving the very real difficulties of characterizing Euclidean complexes and formulating reasonable criteria for a whole sequence of complexes connected by mappings to fit smoothly in $E^{n}$. Beyond this, it is shown that for a simplicial complex to be Euclidean, it is sufficient that its 1-skeleton should be Euclidean.

The author has profited from discussions of this material with Ernest Michael, G. D. Mostow, and Edward Nelson.

1. Coverings. We follow the usual practice of designating a topological space $(X, T)$ by the abbreviation $X$. For a uniform space $(X, \mu)$ we write $\mu X$. As is fairly well known, the uniformity is determined by a knowledge of

(a) which relations in $X$ are entourages, or

(b) which coverings of $X$ are uniform, or

(c) which pseudometrics on $X$ are uniformly continuous. In this

Received August 20, 1957. Supported by a National Science Foundation fellowship. Presented in part to the American Mathematical Society, December 27, 1956, under the title "Weak uniformities induced by families of real-valued functions". 
paper we are concerned mostly with coverings, and therefore we adopt the convention that $\mu$ is the family of all uniform coverings. It is convenient to choose the convention according to which a uniform covering need not consist of open sets. Let us recall the defining conditions : $X$ is a completely regular topological space and $\mu$ is a family of coverings of the set $X$ such that

(i) If $u \in \mu$ and $u$ is a refinement of $v$, then $v \in \mu$;

(ii) The intersection $u \wedge v$ of two coverings in $\mu$ is in $\mu$;

(iii) Every covering in $\mu$ has a star-refinement in $\mu$;

(iv) If $\left\{U_{\alpha}\right\} \in \mu$ then the interiors $U_{\alpha}{ }^{0}$ form a covering and this covering is in $\mu$;

(v) For any point $x$ the stars of $x$ with respect to coverings in $\mu$ form a neighborhood basis at $x$. (The reader who is unfamiliar with the terminology should consult Tukey. [10])

Recall the notation $u<^{*} v$ for " $u$ is a star-refinement of $v$ ", and $S t(A, u)$ for the star of a set $A$ with respect to a covering $u$. A normal sequence of coverings is a sequence $\left(u^{n}\right)$ satisfying $u^{n+1 *}<u^{n}$ for all $n$. Recall that a function is uniformly continuous if and only if the inverse image of every uniform covering is uniform.

We need the fundamental result

1.0. For every uniform covering $u$ of a uniform space $\mu X$ there is a uniformly continuous pseudometric $d$ on $\mu X$ such that for each $x$ in $X$, the set of all $y$ such that $d(x, y)<1$ is a subset of some element of $u$. Exactly this result does not seem to be in print, though Bourbaki has a proof [3] of the corresponding statement connecting entourages with pseudometrics. It will suffice to sketch the similar proof of 1.0. Take a normal sequence $\left(u^{n}\right)$ of uniform coverings, with $u^{0}=u$. For each $x$, $y$, in $X$, let $g(x, y)$ be 0 if $S t\left(x, u^{n}\right)$ contains $y$ for all $n, 2$ if $S t\left(x, u^{n}\right)$ never contains $y$, and otherwise $2^{1-n}$, where $n$ is the largest index for which $y \in S t\left(x, u^{n}\right)$. Let $d(x, y)$ be the infimum of 1 and all the various finite sums $\sum g\left(p_{i}, p_{i+1}\right)$, where $p_{1}=x$ and $p_{n}=y$. By the form of the definition, $d$ is a pseudometric. To see that $d$ is uniformly continuous on $\mu X \times \mu X$, it suffices to observe that $u^{n} \times u^{n}$ is a uniform covering on each element of which $d$ varies no more than $2^{1-n}$. Finally, suppose $\sum g\left(p_{i}, p_{i+1}\right) \leqq 1, p_{1}=x, p_{n}=y$. If we pick $p$ and $q$ respectively so that

(1) $p$ is the last $p_{i}$ such that $g\left(p_{1}, p_{2}\right)+\cdots+g\left(p_{i-1}, p_{i}\right) \leqq 1 / 2$, and

(2) $q$ is the last $p_{j}$ such that $g\left(p_{i}, p_{i+1}\right)+\cdots+g\left(p_{j-1}, p_{j}\right) \leqq 1 / 2$, then computation shows that also $g\left(p_{j}, p_{j+1}\right)+\cdots+g\left(p_{n-1}, p_{n}\right) \leqq 1 / 2$. If $x$ and $y$ are not both in some element of $u^{1}$, then one of the pairs $(x, p),(p, q)$, $(q, y)$, fails to be contained in any element of $u^{2}$. Then induction leads to a contradiction which completes the proof.

A family of functions $f_{a}$ all defined on one uniform space $\mu X$ into 
one uniform space $\nu Y$ is equiuniformly continuous if for each uniform covering $v$ of $\nu Y$ there is a uniform covering $u$ of $\mu X$ which is at once a refinement of all $f_{a}^{-1}(v)$. We wish to regard the nerve of a covering, or any simplicial complex, as a uniform space in the structure in which a mapping $f$ into the complex is uniformly continuous if and only if the functions $f_{\alpha}$, into the real line, which are the barycentric coordinates of $f$, form an equiuniformly continuous family. This dictates the following definition. A uniform complex $\mu X$ is a simplicial complex $X$ consisting of points $x$ with barycentric coordinates $x_{\alpha}$, provided with the distance function $d(x, y)=\max \left|x_{\alpha}-y_{\alpha}\right|$, and the uniformity $\mu$ induced by $d$.

In this paper the nerve $N(u)$ of a covering $u$ is always regarded as a uniform complex. The general vertex of the nerve of $\left\{U_{\alpha}\right\}$ is called $\alpha$. The star of a vertex $\alpha_{0}$ is the union of the incident simplexes, that is, the set of all points $q$ in $N\left(\left\{U_{\alpha}\right\}\right)$ with nonzero $\alpha_{0}$ th coordinate. Note that the stars of vertices always form an open covering $\{S t(\alpha)\}$, but this covering is uniform if and only if the complex is finite-dimensional. For any function $h$ with values in a uniform complex, the coordinate functions $h_{\alpha}$ constitute a partition of unity. If $\left\{h_{\alpha}\right\}$ is a partition of unity subordinated to the covering $\left\{U_{\alpha}\right\}$, this means precisely that for all $\alpha$, $h^{-1}(S t(\alpha)) \subset U_{\alpha}$. In the finite-dimensional case we may summarise as follows. A covering $w$ is realized by a mapping into a uniform space if it is refined by the inverse image of some uniform covering. We have

1.1. An equiuniformly continuous partition of unity subordinated to a finite-dimensional uniform covering of a uniform space determines a realization of the covering by a uniformly continuous mapping into its nerve.

It should be noted that for infinite-dimensional complexes it might well be desirable to employ a different uniformity, and perhaps even a different topology. In this paper we shall be concerned only with finitedimensional complexes, and the choice of definitions is partially justified by

1.2. TheOREM. To every finite-dimensional uniform covering of a uniform space there is subordinated an equiuniformly continuous partition of unity.

Proof. For every uniform covering $u$ of $\mu X$ there is a uniformly continuous pseudometric $d$, as given by 1.0 , such that each point $x$ is in at least one $U_{\alpha} \in u$ which contains the sphere of $d$-radius 1 about $x$. If $u$ is finite-dimensional, so that each $x$ is in at most $n$ sets $U_{\alpha}$, consider the functions $d_{x}(x)=d\left(x, Y-U_{\alpha}\right)$. For each $x, \sum d_{\alpha}(x)$ is a 
finite sum and hence a definite real number $e(x) \geqq 1$. Let $f_{\alpha}(x)=d_{x}(x)$ $l e(x)$. The functions $f_{\alpha}$ form a partition of unity subordinated to $u$. For any $\varepsilon>0$, the covering of $X$ consisting of all apheres of $d$-radius $\varepsilon$ is uniform; and on such a sphere no $f_{\alpha}$ varies more than $4 n \varepsilon$ (by a computation). Thus $\left\{f_{\alpha}\right\}$ is equiuniformly continuous.

It follows, of course, that a uniform covering can be realized by a mapping into a Euclidean space if its nerve is uniformly equivalent to a subspace of a Euclidean space. Let us call such a uniform complex a Euclidean complex, and such a covering a Euclidean covering.

Smirnov has defined [9] a " uniform complex" as a geometric complex $K$ in a Euclidean space $E^{n}$ such that the diameters of the simplexes of $K$ are bounded above and the distances between pairs of disjoint simplexes of $K$ are bounded away from zero. Because of the overlapping terminology, it should be observed that an abstract complex $K$ is Euclidean, as defined above, if and only if it can be embedded in some $E^{n}$ as a uniform complex in the sense of Smirnov. The proof of "if" is trivial; the converse is an exercise which we may omit, since it will follow from 1.8 .

A covering $u$ is star-bounded, of density at most $n$, if each element of $u$ meets at most $n$ other elements of $u$. (The term "star-bounded" is due to Mostow [8], "density" to Boltyanski [1].) Obviously a starbounded covering is star-finite and finite-dimensional, but not conversely. A collection $v$ of sets is said to be discrete relative to a covering $u$ if no element of $u$ meets two different elements of $v$. (Note that a subspace of $\mu X$ is discrete in the induced uniformity if and only if it is a discrete collection of points relative to some covering in $\mu$.) A covering $u$ may be a finite union of collections, $u^{1}, u^{2}, \cdots$, each of which is discrete relative to $u$. Clearly such a covering is starbounded; conversely.

1.3. Every star-bounded covering $u$ is the union of finitely many subcollections each of which is discrete relative to $u$.

Proof. In $u=\left\{U_{\alpha}\right\}$ let $\left\{U_{\beta}^{1}\right\}$ be a maximal subset such that no set $U_{\alpha}$ meets more than one $U_{\beta}^{1}$. Evidently $\left\{G_{\beta}^{\prime}\right\}$ is discrete relative to $u$. Now in $\left\{U_{\alpha}\right\}$, for each $U_{0}$, there are at most $m$ sets $U_{\alpha}$ meeting $U_{0}$, and each of these meets at most $m-1$ more sets $U_{\gamma}$; let this family of $1+m+\left(m^{2}-m\right)$ or fewer sets be called $\mathrm{F}_{0}$. Each $F_{a}$ meets $\left\{U_{\beta}^{1}\right\}$, since otherwise $U_{\alpha}$ could be added to the supposedly maximal family. Having $u^{1}=\left\{U_{\beta}^{1}\right\}, u^{2}, \cdots, u^{k}$, let $u^{k+1}$ be a maximal subset of $\left\{U_{\alpha}\right\}$ disjoint from $u^{1}, \cdots, u^{k}$, and such that no element of $\left\{U_{a}\right\}$ meets more than one element of $n^{k+1}$. For each $U_{\alpha}$ which is not in $u^{1}, \cdots, u^{k}$, necessarily $u^{k+1}$ meets $F_{a}$ (as above). Therefore if $U_{\alpha}$ is not in $u^{1}, \cdots, u^{m^{2}}$, then $F_{a}$ is exhausted and $U_{\alpha}$ is in $u^{m^{2}+1}$. 
REMARK. The properties just shown to be equivalent are graphtheoretic, that is, they depend only on the 1-skeleton of the nerve of the covering.

Tukey has defined a star-finite collection of coverings as a collection, the union of any two of whose members is star-finite [9]. He proved (though he states less) that a uniform star-finite covering has a uniform star-refinement such that the union of the two coverings is star-finite, and hence by induction one has a normal sequence which is a star-finite collection [10, pp. 49-50]. Similarly we define a star-bounded collection of coverings as a collection, the union of any two of whose members is star-bounded; the corresponding result is given below (1.6).

A Euclidean covering is star-bounded, and more. Let us say that the covering $u$ is of polynomial growth if there is a real polynomial $P$ such that, for each $U \in u$, for all natural numbers $k$, the number of elements $V$ of $u$ such that there is a chain $U=U_{0}, U_{1}, \cdots, U_{k}=V$, all $U_{i}$ in $u, U_{i} \cap U_{i+1}$ nonempty for all $i$, is bounded by $P(k)$.

\subsection{Every Euclidean covering is of polynomial growth.}

Proof. Suppose the nerve $N(u)$ is embedded in $E^{n}$ by a uniform equivalence. Let $d$ be the distance function in $N(u)$ and $e(x, y)$ the Euclidean distance between the images of $x$ and $y$. There is $\varepsilon>0$ such that $d(x, y) \geqq 1$ implies $e(x, y) \geqq \varepsilon$; and there is $\delta>0$ such that $d(x, y) \leqq \delta$ implies $e(x, y) \leqq 1$. If $x$ and $y$ are vertices of $N(u)$ corresponding to members of $u$ which are joined by a chain of length $k$, then $e(x, y) \leqq k / \delta$. Then for each vertex $x$, the set of all such $y$ is a set of points whose mutual $e$-distances are all at least $\varepsilon$, packed in a Euclidean sphere of radius $k / \delta$; hence their number is bounded by a polynomial in $k$.

Call a covering linear if its nerve is uniformly equivalent to a subspace of the real line $R$.

1.5. A covering $u$ is linear if and only if it can be indexed with integers, $u=\left\{U_{i}\right\}$, so that $U_{m}$ meets $U_{n}$ only if $|n-m| \leqq 1$. This is equivalent to the conditions that $u$ is,

(a) countable,

(b) one-dimensional,

(c) acyclic,

(d) atriodic, that is, of density 2 or less, and;

(e): ( i ) the nerve of $u$ does not contain three disjoint half-lines ;

(ii) if the nerve contains a whole line then it is connected;

(iii) if the nerve contains two disjoint half-lines then it has only finitely many components.

The proof is omitted. Note that connectedness implies (e). 
By a standard argument (cf. 1.1 of [4]) we obtain

1.6. Let the covering $v$ be a star-refinement of $u$, that is, $v<* u$. If $u$ is

(a) star-bounded, or.

(b) of polynomial growth, or.

(c) linear, then there exists a covering $w$ which also satisfies (a), (b), or (c) such that $v<w^{*}<u$. Further, $u \cup w$ is star-bounded; thus if $u$ is a uniform star-bounded covering of a uniform space then there is a star-bounded normal sequence of uniform coverings $u^{n}$ such that $u^{1}=u$.

Proof. Let $C$ be the set of all subsets $\gamma$ of $u$ such that there is at least one point common to all the members of $\gamma$. For each ordered pair $(\gamma, \delta)$ of elements of $C$, let $W_{\gamma \delta}$ be the union of all $V \in v$ such that the set of all elements of $u$ which contain $V$ is precisely $\gamma$, and the set of all elements of $u$ which contain $S t(V, v)$ is precisely $\delta$. Let $w=\left\{W_{\gamma_{\delta}}\right\}$.

Clearly $v<w$. For any nonempty $W_{\gamma \delta}, \delta$ is nonempty, and any $V$ which meets $W_{\gamma \delta}$ is contained in every member of $\delta$. Thus $\operatorname{St}\left(W_{\gamma \delta}, w\right) \subset U$ for any member $U$ of $\delta$, and $w<* u$.

If $u$ is star-bounded of density $\mathrm{m}$, then for each $W_{\gamma \delta}$ choose $U \in \delta$. No $W_{\alpha \beta}$ can meet $W_{\gamma \delta}$ unless every element of $\alpha$ and of $\beta$ meets $U$; therefore there are at most $2^{2 m}$ such $W_{\alpha \beta}$, and $w$ is star-bounded. Clearly $u \cup w$ is star-bounded, and the last statement of the theorem follows by induction.

If the growth of $u$ is bounded by a polynomial $P(n)$, then $u$ is starbounded of density $m \leqq P(1)$, and the growth of $w$ is bounded by $2^{2 m} p$. It may be of interest to note that this is a polynomial of the same degree as $P$.

Now suppose $u$ is linear. We must modify the above covering $\left\{W_{\gamma \delta}\right\}$. Observe that if $W_{\gamma \delta}$ is not empty then each of $\gamma$ and $\delta$ consists of one or two elements. If $u$ is indexed as in $1.5, u=\left\{U_{n}\right\}$, then there are four possibilities:

(a) $\gamma=\delta=\{n\}$, for some $n$;

(b) $\gamma=\{n, n+1\}, \delta=n$;

(c) $\gamma=\delta=\{n, n+1\}$;

(d) $\gamma=\{n, n+1\}, \delta=\{n+1\}$. For each $n$, replace the two sets described under (b) and (c) with their union. One readily verifies that the modified $w$ is a linear covering satisfying $v<w<* u$.

From 1.6 we may deduce that, for any uniformity $\mu$, the set of all star-bounded coverings in $\mu$ forms a basis for a uniformity, say $b \mu$. The axioms on coarsening (i), intersection (ii), and interiors (iv) are obvious; star-refinement (iii) follows from 1.6, and the neighborhood basis axiom (v) from the fact that every finite covering is star-bounded. 
Since the inverse image of a star-bounded covering, under any function, is star-bounded, therefore when $f: \mu X \rightarrow \gamma Y$ is uniformly continuous, $f: b \mu X \rightarrow b \gamma Y$ is also uniformly continuous. We summarize this (as in [4]) in the slightly elliptical statement that $b$ is a functor. All this is true also for coverings of polynomial growth. However, linear coverings do not in general suffice, for the set of all linear coverings in $\mu$ is not closed under finite intersection. The finite intersections of linear coverings in $\mu$ do form a basis for a uniformity, which is the familiar uniformity $c \mu$ induced by real-valued uniformly continuous functions. To see this it suffices to observe that, by 1.2 and 1.1 , to every linear uniform covering $u$ one may asssciate a mapping into $N(u) \subset R$ which realizes $u$.

1.7. For any uniform space $\mu X$, the star-bounded coverings in $\mu$, as well as those of polynomial growth, form a basis for a uniformity consistent with the topology. Both of these transformations are functors. The weak uniformity c $\mu$ induced by the real-valued uniformly continuous functions on $\mu X$ has a basis consisting of all the Euclidean coverings in $\mu$, and $a$ sub-basis consisting of all the linear coverings in $\mu$.

The proof that Euclidean coverings from a basis for $c \mu$ is again by 1.2 and 1.1. Whether any purely combinatorial result such as 1.6 is valid for Euclidean coverings is not known. (Of course 1.6 applies if it is true that every countable covering of polynomial growth is Euclidean.)

Let $m E^{n}$ denote Euclidean $n$-space, $m R$ the line, in the usual uniformity. Note that $m E^{n}$ is the product of $n$ copies of $m R$. Beyond this we may omit the " $m$ " for the present, since no other uniformities on these spaces are being considered.

1.8. Theorem. A necessary and sufficient condition that a uniform complex $X$ be Euclidean is that the vertices of $X$ may be identified with a set of points in some $E^{n}$, any two of which are at distance greater than 1, so that the distances bctween pairs of vertices which are joined by an edge (1-simplex) of $X$ are bounded. In fact, this is the necessary and sufficient condition that there exist a uniform equivalence $\varphi$ of $X$ into the product of $E^{n}$ and a cell of some dimension; and $\varphi$ may be taken to be semilinear.

Proof. The necessity (both statements) is evident. Suppose conversely that $f$ maps the vertices $\alpha$ of $X$ into $E^{n}$, with the distance from $f(\alpha)$ to $f(\beta)$ greater than 1 for all $\alpha \neq \beta$, and less than $M$ when $\alpha$ and $\beta$ are joined by an edge. For any $x=\left(x_{\alpha}\right)$ in $X$, define $\left(\varphi_{1}(x), \cdots\right.$, $\left.\varphi_{n}(x)\right)=g(x)=\sum x_{x} f(\alpha) \in E^{n}$. Evidently $g$ is uniformly continuous. 
Let $C_{\alpha}$ be the sphere of radius $2 M+1$ about $g(\alpha)$; let $K_{\alpha}$ be the least subcomplex of $X$ which contains $g^{-1}\left(C_{\alpha}\right)$. The vertices of $K_{\alpha}$ are mapped by $f$ into points of distance 1 or more from each other in a sphere of radius $3 M+1$, and hence their number has a bound $q+1$. Then each $K_{\alpha}$ may be embedded by an isometry $k_{\alpha}$ in the abstract $q$ dimensional simplex; embedding the simplex in a cell in $E^{q}$, we obtain mappings $h_{\alpha}: K_{\alpha} \rightarrow E^{q}$ which are semilinear uniform equivalences, having a common modulus of continuity, and such that the mappings $h_{\alpha}{ }^{-1}$ have a common modulus of continuity. Define an extension $i_{\alpha}$ of $h_{\alpha}$ over $X$ as follows: every $x$ in $X$ can be expressed uniquely as a convex combination $t y+(1-t) z$, where $y$ is in the subcomplex $K_{a}$ and $z$ has coordinate $z_{\beta}=0$ for all $\beta$ in $K_{\alpha}$; let $i_{\alpha}(x)=t h_{\alpha}(y)$. Then $\left\{i_{\alpha}\right\}$ is an equiuniformly continuous family of semilinear mappings. Further, there is a cell in $E^{q}$ which contains all their ranges.

Finally, $\left\{g\left(K_{\alpha}\right)\right\}$ is a star-bounded covering of $g(X)$, and thus, by 1.3 , it is a union of subcollections $v^{1}, \cdots, v^{s}$, each discrete relative to the whole covering. For $j=1, \cdots, s$, let $d_{j}=\sum\left[i_{\alpha} \mid g\left(K_{\alpha}\right) \in v^{j}\right]$. Observe that on each star $S t(\beta)$ in $X, d_{j}$ coincides with one $i_{\alpha}$ (that is, at most one fails to vanish; for $S t(\beta) \subset K_{\beta}$, and $g\left(K_{\beta}\right)$ meets at most one $g\left(K_{\alpha}\right)$ in $v^{\jmath}$ ). Therefore $d_{j}: X \rightarrow E^{q}$ is uniformly continuous. The definition of $\varphi: X \rightarrow E^{n+q s}$ is completed by putting $\left(\varphi_{k}(x)\right), n+(j-1) q+1 \leqq k \leqq n+j q$, equal to the vector $d_{j}(x)$, for each $j=1, \cdots, s$.

We have a uniformly continuous semilinear mapping $\varphi$ of $X$ into the product of $E^{n}$ and a $q s$-dimensional cell. Uniform continuity of $\varphi^{-1}$ means that for each $\varepsilon>0$ there is $\delta>0$ such that two points at distance $>\varepsilon$ in $X$ are mapped by $\varphi$ into points at distance $>\delta$. For any two points, $x, y$, in $X$, either $g$ maps them into points at distance $>1$ (and so does $\varphi$ ), or they lie in a common $K_{\alpha}$. But then some $d_{j}$ coincides on $K_{\alpha}$ with the embedding $h_{\alpha}$. Thus $\varphi$ is a uniform equivalence.

1.9. CoRollary. If the 1-skelton of a uniform complex $X$ is Euclidean then $X$ is Euclidean.

1.10. Theorem. The following conditions on a uniform complex $X$ are equivalent.

(a) $X$ is a countable, star-finite, finite-dimensional complex.

(b) $X$ is a locally compact, $\sigma$-compact, finite-dimensional space.

(c) $X$ is homeomorphic with a closed subset of a Euclidean space.

(d) There is a distance-increasing homeomorphism of $X$ into a Euclidean space.

(e) There is a uniformly continuous homeomorphism of $X$ upon a closed subset of a Euclidean space. 
Proof: The implications $(\mathrm{e}) \Rightarrow(\mathrm{c}),(\mathrm{d}) \Rightarrow(\mathrm{c})$, and $(\mathrm{c}) \Rightarrow(\mathrm{b}) \Rightarrow(\mathrm{a})$, are evident. From the hypothesis (a) that $X$ is a countable star-finite uniform complex of dimension $n$, we shall construct the mappings of $X$ into $E^{2 n+3}$ required for (d) and (e). Clearly it suffices to construct such a mapping into $E^{2 n+2}$ for each component of $X$. Let $Y$ be a component of $X$, and let $\varphi$ be a one-to-one semilinear mapping of $Y$ into $E^{2 n+1}$. (To construct $\varphi$ it suffices to map the vertices of $Y$ upon a set of points in general position in $E^{2 n+1}$.) Since $Y$ is star-finite, $\varphi$ is continuous.

Choose a vertex $Y_{0}$ of the complex $Y$ and let $f_{0}$ be a one-to-one semilinear mapping of $Y$ into $E^{2 n+2}$ which sends $Y_{0}$ to the origin and all of $Y$ into the hyperplane $x_{1}=0$. Let $Y_{1}$ be the subcomplex which is the closure of the star of $Y_{0}$; inductively let $Y_{k+1}$ be the subcomplex spanned by $Y_{k}$ and the vertices which are joined to $Y_{k}$ by 1-cells of $Y$. Since $Y$ is star-finite, each $Y_{k}$ is a finite complex; and since $Y$ is connected, the union of all $Y_{k}$ is $Y$. Let $Z_{k}$ be the span of the vertices not in $Y_{k}$. For each $k$, each point $p$ of $Y$ can be written uniquely (in barycentric coordinates) as $\lambda p_{1}+(1-\lambda) p_{2}$, with $\lambda$ and $1-\lambda$ nonnegative, $p_{1}$ in $Y_{k}, p_{2}$ in $Z_{k}$. Inductively, let $f_{k}$ be a piecewise linear one-to-one mapping of $Y$ into $E^{2 n+2}$, sending $Y$ into the halfspace $x_{1} \leqq c_{k}$ and $Z_{k-1}$ into the hyperplane $x_{1}=c_{k}$ and increasing distances in $Y_{k}$. Write $f_{k}(p)$ $=g(p)+h(p)$, where $g(p)$ is the projection of $f_{k}(p)$ on the $x_{1}$-axis, $h(p)$ the projection on $x_{1}=0$. For $p$ in $Z_{k}, f_{k+1}(p)$ is to be $\alpha g(p)+\beta h(p)$, where $\alpha$ and $\beta$ are large constants to be determined. For $p$ in $Y_{k}, f_{k+1}(p)$ $=f_{k}(p)$; and for general $p=\lambda p_{1}+(1-\lambda) p_{2}$ (as above), $f_{k+1}(p)$ must be $\lambda f_{k+1}\left(p_{1}\right)+(1-\lambda) f_{k+1}\left(p_{2}\right)$. On $Z_{k}, g$ is constant, and $h$ is one-to-one, piecewise linear, and continuous. The common part of $Z_{k}$ and $Y_{k+1}$ is a finite complex, and hence there exists $\beta$ so large that $\beta h$ increases distances on this complex. Similarly, if $\alpha$ and $\beta$ are large enough, $f_{k+1}$ will increase distances on $Y_{k+1}$, and the induction runs. Finally we have a sequence $\left(f_{k}\right)$ of continuous mappings of $Y$ into $E^{2 n+2}$, converging locally uniformly to a limit $\psi$. Then $\psi$ is continuous ; and $\psi$ increases distances, which implies that $\psi^{-1}$ is continuous. Thus (a) implies (d).

Since each $Y_{k}$ is compact, one can go back and modify the constants $\alpha$ and $\beta$ at each step so as to end with a uniformly continuous homeomorphism $g$ upon an image which is not necessarily a closed set. Define a real-valued function $h$ on $Y$ as follows. For the distinguished vertex $Y_{0}, h\left(Y_{0}\right)=0$. For any other point $y$ there is just one $k$ such that $y$ is in $Y_{k+1}$ but not in $y_{k}$; and there is a unique relation $y=\lambda p_{1}+(1-\lambda) p_{2}$, $p_{1} \in Y_{k}, p_{2} \in Z_{k}$. Let $h(y)=k+1-\lambda$. Evidently $h$ is uniformly continuous. Let $h^{\prime}(y)$ be the point in $E^{2 n+2}$ whose first coordinate is $h(y)$, with all other coordinates zero ; then $g+h^{\prime}$ is a uniformly continuous homeomorphism upon a closed set. This completes the proof.

The complexes satisfying $(d)$ (in slightly different words) are called 
Lebesgue complexes by Smirnov. [9] Evidently in any fixed $E^{n},(d)$ and (e) are not equivalent (if $n>1$ ).

2. Bases. This section is primarily a discussion of the subspaces of the line $m R$, including a characterization; it concludes with a formulation of the same approach to subspaces of $m E^{n}$.

Let us first suppose given the topological space $R$, and characterize $m$ among its uniformities. Evidently $m$ is

(a) metric, that is, it has a countable basis of coverings. It has

(b) a star-bounded basis, and it is

(c) uniformly locally connected, that is, there is a basis of coverings whose elements are connected sets. We shall see that these properties are shared by $m$ only with the uniformities induced by metrizing $R$ as $(0,1)$ or as a half-infinite interval; thus $m$ can be characterized by adding the condition $(d)$ : the space is complete.

These are evidently not the conditions to apply to subspaces of $m R$, (c) being invalid. We shall have to replace (c) with some sort of conditions on the nerves of the coverings. It is not enough to say $\left(c^{\prime}\right)$ there is a basis of linear coverings, even on the topological space $R$. This is shown by the following subspace of $m E^{2}$. Take the half-line consisting of all points $(x, 0), x \leqq 3$, and for $n=3,4, \cdots$, take the four line segments running successively from $(n, 0)$ to $(n+1-3 / n, 1)$ to $(n$ $+1-2 / n, 0)$ to $(n+1-1 / n, 1)$ to $(n+1,0)$. A sketch shows that this metric space satisfies conditions (a), (b), $\left(c^{\prime}\right)$, and $(d)$, but not (c); it is homeomorphic but not uniformly equivalent to $m R$.

We have indicated some uniformities on $R$ satisfying (a), (b), and (c), but not (d). For (a), (c), and (d), consider the following distance function $f$. For notational convenience let $e$ indicate $e(x, y)=|x-y|$; let $\min (x, y)=m$. If $e \geqq 1$, or if $m \leqq 1$, then $f(x, y)=e$; otherwise $f(x$, $y)=e^{1 / m}$. Finally, to construct a nonmetric uniformity on $R$ satisfying (b), (c), and (d), let $\left(a_{n}\right)$ designate a (variable) sequence of positive numbers coverging to zero. For each natural number $m$, define the covering $u\left(m,\left(a_{n}\right)\right)$ to consist of the following intervals.

(1) For every integer $t$ such that neither $t-1, t$, nor $t+1$ is a positive integral multiple of $m$, the interval $(t-1 / m, t+1 / m)$.

(2) For each positive integer $n$, the intervals $\left(n+a_{n}, n+2 / m\right)$ and $\left(n-2 / m, n-a_{n}\right)$.

(3) For $-m \leqq t \leqq m$, and for all $n$, the intervals $\left(n+(t-1 / m) a_{n}, n\right.$ $\left.+(t+1 / m) a_{n}\right)$. Consider the collection of all $u\left(m,\left(a_{n}\right)\right)$ such that $m \geqq 4$ and $a_{n}<1 / m+1$ for all $n$. One readily verifies that this collection is a basis of a uniformity having the required properties. One may note also that all the above examples have bases consisting of linear coverings. 
2.1. Every uniformly locally connected metric space which is homeomorphic to the real line $R$ and has a star-bounded basis of uniform coverings is uniformly equivalent either to $m R$ or to an open interval of $m R$.

Proof. We may call the space $\mu R$; it is required to construct a uniform equivalence of $\mu R$ into $m R$. We are given a countable basis $\left\{u^{n}\right\}$ for $\mu$, a star-bounded basis $\left\{v^{\alpha}\right\}$, and also a basis consisting of coverings with connected elements. The interiors of these connected sets are open intervals, still forming uniform coverings, which still constitute a basis $\left\{w^{\beta}\right\}$. If for each $n$ we choose $w^{n}$ refining $u^{n}$, we have a countable basis of coverings with open intervals. Evidently we may suppose each $w^{n+1}$ is a star-refinement of $w^{n}$ (since some $w^{n+k}$ is), and we may suppose $w^{1}<v^{\alpha}$ for some $\alpha$. Next we interpolate linear coverings $z^{n}$ consisting of open intervals, $w^{n+1}<z^{n}<w^{n}$, as follows. Choose a point $p$ and let $Z_{0}^{n}$ be $S t\left(p, w^{n+1}\right)$. Evidently $Z_{0}^{n}$ is an open interval $\left(p_{1}, q_{1}\right)$. Having points $p_{k}$ and $q_{k}$, define $Z_{k}^{n}$ as $S t\left(q_{k}, w^{n+1}\right)$ and $Z_{-k}^{n}$ as $S t\left(p_{k}, w^{n+1}\right)$. At some stage an improper interval may be obtained, so that $p_{k}$ or $q_{k}$ does not exist; in that case omit so much of the construction as involves the missing points. Evidently the union of all $Z_{k}^{n}, n$ fixed, $k=0, \pm 1, \cdots$, is a nonempty open and closed subset of $R$, hence all of $R$. Since every intervel in $w^{n+}$ contains at most one of the points $p, p_{k}, q_{k}, w^{n+1}<z^{n}=\left\{Z_{k}^{n}\right\} ;$ and since $w^{n+1}<* w^{n}$, hence $z^{n}<w^{n}$. Clearly $z^{n}$ is linear.

To see that $\left\{z^{n}\right\}$ is a star-bounded family, consider any $m<n$. Each element of $z^{n}$ meets at most two other elements of $z^{n}$ and at most three elements of $z^{m}$. Thus if $z^{m} \mathrm{U} z^{n}$ is not star-bounded then there exist sets $Z$ in $z^{m}$ meeting arbitrarily many elements of $z^{n}$. A fortiori there exist sets $V$ in $v^{\alpha}$ meeting arbitrarily many elements of $z^{n}$. Since $z^{n}$ is linear, one can find for each positive integer $r$ a set $V \in v^{\alpha}$ countaining $r$ points, no two of which lie in a common element of $z^{n}$. But there is a covering $v^{\beta}<z^{n}$ such that $v^{\alpha} \cup v^{\beta}$ is star-bounded, since the $v^{\prime}$ s form a starbounded basis. The contradiction establishes that $z^{m} \cup z^{n}$ is star-bounded ; and the family $\left\{z^{n}\right\}$ is a countable star-bounded basis consisting of linear coverings each of which consists of open intervals.

Now index the elements of $z^{n}$ with rational numbers $s, z^{n}=\left\{Z_{s}^{n}\right\}$, as follows. For $n=1$, the values of $s$ are the integers $k$ assigned above; thus $Z_{s}^{n}$ does not meet $Z_{t}^{n}$ if $|s-t|>1$. Having indexed $z^{n}$, consider each $Z_{s}^{n}$. There is a next rational number $t>s$ such that some element of $z^{n}$ is called $Z_{t}^{n}$, except possibly for one (greatest) value of $s$; if there is such en exceptional $s$, assign to it the value $t=s+2^{-n}$. There are finitely many elements $Z$ of $z^{n+1}$ such that $s$ is the least index such that $Z \subset Z_{s}^{n}$; and the number of them, $h(s)$ is a bounded function of $s(n$ fixed). Furthermore, exactly one of them meets an element of $z^{n+1}$ 
which is contained in the next $Z_{q}^{n}$ before $Z_{s}^{n}$ (with a possible exception if there is no such $q$ ) and exactly one meets an element of $z^{n+1}$ which is contained in $Z_{t}^{n}$ but not in $Z_{s}^{n}$. Index these elements of $z^{n+1}$ in order from $Z_{q}^{n}$ toward $Z_{t}^{n}$ as $Z_{i}^{n+1}$, for $i=s, s+(t-s / h(s)$ ), $\cdots, t-(t-s) / h(s)$ (equal steps). This completes the indexing. Then routine computation shows that for each point $x$ in $\mu R$, the numbers $g_{n}(x)=\max \left[s \mid x \in Z_{s}^{n}\right]$ converge to a limit $g(x)$, and that $g$ is a uniformly continuous function realizing all of the coverings $z^{n}$. Since $\left\{z^{n}\right\}$ is a basis, $g$ is one-to-one and $g$ is a uniform equivalence.

If one tries to carry out the construction of 2.1 on the example given previously of a complete metric space homeomorphic to $R$ having a star-bounded basis of linear uniform coverings, it breaks down because ultimately $z^{n+1}$ must be "crooked" in $z^{n}$. It is not crooked in the strong sense familiar from the construction of the pseudo-arc; indeed, with a suitable choice, one can arrange that near any point in the space almost all $z^{n+1}$ are "straight" in $z^{n}$. Up to some critical value $N$ the chains $z^{n}$ follow an approximating smooth path; then $z^{N+1}$ and all subsequent $z^{n}$ follow the kinds in the curve. This means that we must impose a very strong straightness condition in order to characterize the subspaces of $m R$. Let us use the term chain in $u$ for a subset of a covering $u$ whose elements correspond to the vertices of a chain of edges in $N(u)$.

2.2. The following conditions on a uniform space $\mu X$ are necessary and sufficient in order that $\mu X$ should be uniformly equivalent to a subspace of $m R$.

(a) $\mu$ has a basis which is a countable sequence of linear coverings $u^{n}$, with $u^{n+1}<u^{n}$, such that (1) if $\left(U_{1}, \cdots, U_{p}\right)$ is a chain in $u^{n+1}$, with $U_{1}$ and $U_{p}$ both contained in one element $U$ of $u^{n}$, then all $U_{i}$ are contained in $U$; and $(2)$ if $\left(U_{1}, \cdots, U_{p}\right)$ and $\left(V_{1}, \cdots, V_{q}\right)$ are two chains in $u^{n+1}$ having no common elements, some element $U$ of $u^{n}$ contains both $U_{1}$ and $V_{1}$, and some element $V$ of $u^{n}$ contains both $U_{p}$ and $V_{q}$, then $U$ meets $V$.

(b) $\mu$ has a star-bounded basis.

The necessity of the conditions is obvious, and the proof of sufficiency is an easy modification of 2.1. However, the proof as given above does not look ready to be generalized to $E^{n}$. We conclude this section with some easily proved remarks outlining another version which might have brighter prospects.

First, it suffices to work with the completion. Second, if a complete uniform space has a countable basis consisting of finite-dimensional coverings, (a) there is a natural inverse system of semilinear mappings on the nerves of these coverings, and (b) the space is the inverse limit of this system. I have in mind the mappings defined, for a sequence $\left\{u^{n}\right\}, u^{n+1}<^{*} u^{n}$, as follows. Since $u^{n}$ is finite-dimensional, each element 
$U_{\alpha}$ of $u^{n+1}$ is contained in only finitely many elements of $u^{n}$, and the corresponding vertices in $N\left(u^{n}\right)$ span a simplex; the vertex $\alpha$ of $N\left(u^{n+1}\right)$ can be taken to the center of gravity of that simplex, in a uniformly continuous semilinear mapping. Third, if all the nerves can be embedded in one complete space in such a way that the mappings $N\left(u^{n+1}\right) \rightarrow N\left(u^{n}\right)$ move no point more than $\varepsilon_{n}$, where $\varepsilon_{n} \rightarrow 0$, then of course the inverse limit space is embedded in the same containing space. This is clearly possible under the hypotheses of 2.2 .

3. The weak derivative. In this section we describe an operation on uniformities which generalizes the passage from the usual uniformity $m$ on a Euclidean space to the finest uniformity $a$. It is not known whether this operation is applicable to general uniformities ${ }^{1}$; the main results of this section apply only to weak uniformities induced by families of real-valued functions.

For any weak uniformity $\mu$ on a space $X$, we define the weak $d e$ rivative $w \mu$ of $\mu$ as the family of all coverings of $X$ which have a refinement of the form $\left\{U^{\alpha} \cap V_{i}^{\alpha}\right\}$, where $\left\{U^{\alpha}\right\}$ is a covering in $\mu$ and the families $V^{\alpha}=\left\{V_{i}^{\alpha}\right\}$, for each $\alpha$, are finite coverings in $\mu$ of bounded dimension. (This is a modification of an operation called the derivative in [4]. We might as well have required $v^{\alpha}$ only to cover the subspace $U_{\alpha}$; the equivalence follows from the simple proposition 3.6 below.) If we recall that since $\mu$ is a weak uniformity, the covering $\left\{U_{\alpha}\right\}$ may be supposed Euclidean, we see that the typical covering $\left\{U_{\alpha} \cap V_{i}^{\alpha}\right\}$ is (1) uniformly locally uniform (on $\mu X$ ), (2) uniformly locally finite, and (3) finite-dimensional.

The proof that $w \mu$ is a uniformity will be a demonstration that $w \mu$ is the weak uniformity induced by a certain family of functions. Let $C(\mu X)$ denote the family of all real-valued uniformly continuous functions on $\mu X$ (uniformly continuous into $m R$ ). The term composition will be used with the specific meaning of a functional composition $g\left(f_{1} \cdots, f_{n}\right)$, where $f_{1}, \cdots, f_{n}$, are in $C(\mu X)$ and $g$ is any continuous real-valued function on $E^{n}$. In particular, the family of all such functions on $X$ to $R$ is the closure, under composition, of $C(\mu X)$. (Cf. [5].)

3.1. For each Euclidean space $E^{n}$, the weak derivative of the usual metric uniformity, $m$, is the finest uniformity consistent with the topology;

1. Specifically, applying the definition of $w_{\mu}$ in the next paragraph to a general uniformity $\mu$, it is not known wheter $w \mu$ is always a uniformity in the present sense. The referee points out that it is certainly a regular uniformity in the sense of Morita and [7]; and there is a non-trivial theory of such structures. In that theory, the referee observes, 3.5 is valid without restriction on $\mu$. 
that is, the uniformity a defined by all open coverings.

Proof. Evidently any covering in $w m$ has an open refinement. Conversely, for any open covering $\left\{W_{\gamma}\right\}$ of $E^{n}$, consider a uniform covering $\left\{U_{x}\right\}$ consisting of closed metric spheres. Since each $U_{a}$ is a compact space, there is a finite uniform covering $\left\{G_{i}^{\alpha}\right\}$ of $U_{\alpha}$ refining the open covering $\left\{U_{\alpha} \cap W_{\gamma} \mid\right.$ all $\gamma_{j}$. We may take $\left\{G_{i}^{\alpha}\right\}$ n-dimensional. Let $p_{\alpha}$ be the center of the sphere $U_{\alpha}$, and for each $G_{i}^{\alpha}$ meeting the boundary $B$ of $U_{\alpha}$, let $V_{i}^{\alpha}$ consist of $G_{i}^{\alpha}$ together with all points $q$ outside $U_{\alpha}$ such that the intersection of the segment $p q$ with $B$ is a point of $G_{i}^{\alpha}$; otherwise let $V_{i}^{\alpha}=G_{i}^{\alpha}$. Evidently $\left\{V_{i}^{\alpha}\right\}$ is a uniform finite $n$-dimensional covering of $m E^{n}$, and $\left\{U_{\alpha} \cap V_{i}^{\alpha}\right\}$ is a refinement of $\left\{W_{\gamma}\right\}$. Thus $w m E^{n}$ $=a E^{n}$.

3.2. For any open covering $u$ of a Euclidean space $E^{n}$, there is a homeomorphism of $E^{n}$ onto itself which takes $u$ onto a uniform covering of $m E^{n}$.

This is obvious.

3.3. THEOREM. The weak derivative of a weak uniformity is a weak uniformity. Specifically, if $\mu$ is weak, then $w \mu$ is the weak uniformity induced by the closure under composition of $C(\mu X)$.

Proof. If $f_{1}, \cdots, f_{n}$, are in $C(\mu X), g: E^{n} \rightarrow R$ is continuous, and $u$ is any uniform (even any open) covering of $R$, then the inverse image of $u$ under $g$ is uniform in $a E^{n}=w m E^{n}$ and hence the inverse image of $u$ under $g\left(f_{1}, \cdots, f_{n}\right)$ is in the family of coverings $w \mu^{\cdot}$ Evidently $w \mu$ is closed under intersection; therefore $w \mu$ contains the weak uniformity induced by the closure under composition of $C(\mu X)$.

Conversely, since $\mu$ is weak, each covering $\left\{U_{\alpha} \cap V_{i}^{\alpha}\right\}$ in $w \mu$ may be refined by a covering $\left\{U_{j} \cap V_{i}^{j}\right\}$ so that the following is true. There is a uniformly continuous function $f: \mu X \rightarrow m E^{n}$ realizing $\left\{U_{j}\right\}$. Each $v^{j}$ $=\left\{V_{i}^{j}\right\}$ is finite and at most $k$-dimensional and is realized by a bounded uniformly continuous function $g_{j}: \mu X \rightarrow m E^{q}$ (here $q=2 k+1$ ). Also, $\left\{U_{j}\right\}$ is star-bounded and can be written as the union of $p$ relatively discrete subcollections $u^{r}$; and finally, $\left\{U_{j}\right\}$ is countable. We shall construct a mapping $h$ of $\mu X$ into $(n+p q)$-space.

Choose positive numbers $c_{j}$ such that $c_{j}\left|g_{j}(x)\right|<2^{-j}$ for all $x$. For each $j$, let $d_{j}$ be a uniformly continuous real-valued function on $\mu X$ with values in $\left[0, c_{j}\right]$, vanishing outside the star of $U_{j}$ and having the constant value $c_{j}$ on $U_{j}$. For each $x$, define the first $n$ coordinates of $h(x)$ to be the coordinates of $f(x)$. Let the $q$ coordinates of $h(x)$ from the $(n+q(r-1)$ $+1)$ th through the $(n+q r)$ th be $\sum\left[d_{j}(x) g_{j}(x) \mid U_{j} \in n^{r}\right]$. 
Since the series $\sum c_{j}\left\|g_{j}\right\|$ converges (absolutely), $h: \mu X \rightarrow m E^{s}(s=n$ $+p q$ ) is uniformly continuous. It is clear from the construction that $h$ : $X \rightarrow a E^{s}$ realizes $\left\{U_{j} \cap V_{i}^{j}\right\}=z$, that is, $z$ is refined by the inverse image of some open covering. By 3.2, there is a continuous function $T: E^{s} \rightarrow E^{s}$ such that $T(h): \quad X \rightarrow m E^{s}$ realizes $z$. But each coordinate of $T(h)$ is a composition of a continuous coordinate projection of $T$ with the uniformly continuous coordinates of $h$, and the proof is complete.

From an approximation theorem proved in [5, Theorem 1.7] we have

3.4. Corollary. $C(w \mu X)$ contains all the compositions $g\left(f_{1}, \cdots, f_{n}\right)$, $g$ continuous and $f_{i}$ in $C(\mu X)$, and consists of all uniform limits of such compositions.

In [5] there is an example of a family of functions $A$ such that the uniform closure of the closure under composition of $A$ is not itself closed under composition. That example $A$ is not $C(\mu X)$ for any $\mu$, but this is inessential. We describe an example of a uniform space $\mu X$ such that $\mu$ is weak and $w(w \mu) \neq w \mu$, omitting the details of the verification.

Example. Let $X$ be the set of all ordered triples $(i, j, k)$ of positive integers, with the discrete topology. Let $\mu$ be the set of all coverings $u$ of $X$ such that (1) for some $n^{\prime}$, for each $n>n^{\prime}$, there is an element $U_{n}$ of $u$ which contains all $(n, j, k)$; and (2) for each $n\left(\leqq n^{\prime}\right)$, for some $m^{\prime}$, for each $m>m^{\prime}$ there is an element $U_{n m}$ of $u$ which contains all $(n, m, k)$. Observe that $\mu$ has a basis consisting of discrete coverings; thus $\mu$ is weak, and $w \mu$ and $w w \mu$ can be computed without worrying about dimension. One may verify that a covering $u$ is in $w \mu$ if and only if (a) for each $n$ there is $m^{\prime}=m^{\prime}(n)$ such that for each $m$ $>m^{\prime}$ there are finitely many elements of $u$ whose union contains all $(n$, $m, k$ ), and (b) for some $n^{\prime}$, for each $n>n^{\prime}$, (i ) there are finitely many elements of $u$ whose union contains all $(n, j, k)$, and (ii) for each $m$ $>m^{\prime}(n)$ all points $(n, m, k)$ are in one element of $u$. Then $w w \mu$ is determined by the conditions (a) and (b), (i ); in particular, $w w \mu \neq w \mu$.

Powers of $w$ are defined by $w^{\alpha+1}=w w^{\alpha}$; for limit ordinals $\alpha, w^{\alpha} \mu$ is the union of the increasing sequence of families of coverings $w^{\beta} \mu, \beta<\alpha$. Since the uniformities $w^{\alpha} \mu$ are successively finer, there must be an $\alpha$ such that $w^{\alpha+1} \mu=w^{\alpha} \mu$. (By 3.4, the first uncountable ordinal is such an $\alpha$.)

3.5. Applied to uniform spaces with weak uniformities, the weak derivative and all its powers are functors commuting with completion.

Proof. If $f: \mu X \rightarrow \nu Y$ is uniformly continuous then, since $f^{-1}$ preserves finiteness and dimension of coverings, $f: w \mu X \rightarrow w \nu Y$ is uniformly 
continuous; thus $w$ is a functor. If $F$ is a Cauchy filter in $\mu X$ and $\left\{U_{\alpha} \cap V_{i}^{\alpha}\right\}$ a typical covering in $w \mu$, then $F$ contains some $U_{\alpha}$ and, for that $\alpha$, some $V_{i}^{\alpha}$; being a filter, $F$ contains $U_{\alpha} \cap V_{i}^{\alpha}$. Thus the same filters are Cauchy in $\mu$ and in $w \mu$, and the completions $\pi \mu X$ and $\pi w \mu X$ have the same points. Obviously every covering in $w \pi \mu$ is in $\pi w \mu$; the converse is a routine application of Morita's demonstration [7; Lemma 7, Th. 3, Th. 9] that every uniform covering $\left\{V_{\beta}\right\}$ of $\mu X$ can be extended to a uniform covering $\left\{V_{\beta}^{*}\right\}$ of $\pi \mu X$ such that $V_{\beta}=V_{\beta}^{*} \cap X$ and the correspondence $V_{\beta} \leftarrow \rightarrow V_{\beta}^{*}$ preserves the nerve. Thus $\pi w=w \pi$. Therefore if $w^{x}$ is a functor commuting with $\pi$, so is $w^{\alpha+1}$. The proof is completed by the observation that every covering in $w^{x}$, for $\alpha$ a limit ordinal, is already in some $w^{\beta}$ for $\beta<\alpha$.

The next four propositions amount to a closer analysis of the theorem of [5] that if $C(\mu X)$ is closed under composition then for any subspace $Y$ of $X$, in the induced uniformity $\mu^{*}, C\left(\mu^{*} Y\right)$ contains only the restrictions of function in $C(\mu X)$.

3.6. Let $\mu^{*} Y$ be a subspace of $\mu X$, and $\left\{U_{i}\right\}$ a finite uniform covering of $\mu^{*} Y$ of dimension $k$. There is a finite uniform covering $\left\{V_{j}\right\}$ of $\mu X$, of dimension $2 k+1$ or less, such that $\left\{V_{j} \cap Y\right\}$ is a refinement of $\left\{U_{i}\right\}$.

3.7. The weak derivative preserves subspaces; that is, if $\mu^{*} Y$ is a subspace of $\mu X$ (and $\mu$ is weak) then the uniformity induced on $Y$ by $w \mu$ is $w \mu^{*}$.

3.8. If $f$ is a real-valued function on $X, \mu$ a weak uniformity on $X$, and $\left\{U_{\alpha}\right\}$ a uniform covering of $\mu X$ such that on each $U_{\alpha}, f^{\prime}$ is bounded and uniformly continuous, then $f$ is uniformly continuous on $w \mu X$.

3.9. If $\mu^{*} Y$ is a subspace of $\mu X$ ( $\mu$ a weak uniformity) and $f$ a uniformly continuous real-valued function on $\mu^{*} Y$, then $f$ has an extension in $C(w \mu X)$.

Proof of 3.6. This is a corollary of a theorem of Katětov [6]: every bounded real-valued uniformly continuous function on a subspace of any uniform space has a bounded uniformly continuous extension over the whole space. If $\left\{U_{i}\right\}$ is a finite $k$-dimensional covering of $\mu^{*} Y \subset \mu X$, then $\left\{U_{i}\right\}$ cen be realized by a mapping into a compact subset of $E^{* k+1}$; each coordinate can be extended, by Katétov's theorem, and the conclusion follows.

Proof of 3.7. If $\mu^{*} Y$ is a subspace of $\mu X$ and $\left\{U_{\alpha} \cap V_{i}^{\alpha}\right\}$ a typical 
covering in $w \mu$, then $\left\{U_{a} \cap Y\right\}$ is in $\mu^{*}$, the coverings $y^{\alpha}=\left\{V_{i}^{\alpha} \cap Y\right\}$ are finite coverings in $\mu^{*}$ of bounded dimension, and hence $\left\{U_{\alpha} \cap V_{i}^{\alpha} \cap Y\right\}$ is in $w \mu^{*}$. The converse is clear in the light of 3.6 .

Proof of 3.8. If $f$ is bounded and uniformly continuous on each element of the uniform covering $\left\{U_{\alpha}\right\}$, then the inverse image of any uniform covering of $m R$ is refined by a covering $\left\{U_{\alpha} \cap V_{i}^{\alpha}\right\}$, where for each $\alpha,\left\{V_{i}^{\alpha}\right\}$ is a uniform finite 1-dimensional covering of the subspace $U_{\alpha}$. By 3.6, each $\left\{V_{i}^{\alpha}\right\}$ may be extended to a uniform finite 3-dimensional covering of $\mu X$, and hence $f$ is uniformly continuous on $w \mu X$. (Actually, by the method of 3.6, these coverings $\left\{V_{i}^{\alpha}\right\}$ may be extended so as to remain 1-dimensional.)

We may note that the hypothesis that $\mu$ is weak was not needed for these proofs; thus if $w$ can be satisfactorily interpreted for more general spaces, 3.7 and 3.8 will carry over. (Cf. the footnote. ${ }^{1}$ ) The hypothesis will be used for 3.9, though one could avoid it by a use of results of [4]. It should be noted that the proof of 3.9 is almost the same as the proof of a similar extension theorem in [4].

Proof of 3.9. Note first $(*)$ that a function $h$ which is defined on a uniform space $\rho A$ into a uniform space $\sigma B$, and uniformly continuous on each of a finite family of subspaces of $\rho A$ which make up a uniform covering, is uniformly continuous on $\rho A$. Now consider the given hypothesis, $f: \mu^{*} Y \rightarrow m R$ uniformly continuous, $\mu^{*} Y$ a subspace of $\mu X$. Let $V_{n}=f^{-1}((n-1, n+1))$ in $Y$, and let $U_{n}=V_{n} \cup(X-Y)$. Since $\left\{V_{n}\right\}$ is in $\mu^{*}$, therefore $\left\{U_{n}\right\}$ is in $\mu$. Since $\mu$ is weak, $\left\{U_{n}\right\}$ has a countable uniform star-refinement $\left\{W_{i}\right\}=w$.

The function $f$ is defined, in particular, on the subspace $Y \cap S t\left(W_{1}\right.$, $w)$ of the space $S t\left(W_{1}, w\right) \cap\left(Y \cup W_{1}\right)$. On that subspace $f$ is uniformly continuous and, since $S t\left(W_{1}, w\right) \subset U_{n}$ for some $n, f$ is bounded there. By Katětov's theorem [6] there is a bounded uniformly continuous function $g_{1}$ on $S t\left(W_{1}, w\right) n\left(Y \cup W_{1}\right)$ to $m R$, such that $g_{1}$ and $f$ agree on their common domain $Y \cap S t\left(W_{1}, w\right)$. Therefore, by $(*)$, the function $f_{1}$ on $Y \cup W_{1}$ whose values are those of $f$ and of $g_{1}$ is uniformity induced by $\mu)$.

Having extended $f$ to $f_{n}$, defined on the union of $Y$ and $W_{1}, \cdots$, $W_{n}$, uniformly continuous there, and bounded on each $W_{n i}$, one constructs by the same argument an extension $f_{n+1}$ which is defined on $W_{n+1}$ also. By induction one has a well-defined function $\overline{f^{*}}$ extending $f$ over all of $X$. On each $W_{i}, \bar{f}$ agrees with $f_{i}$ and thus is bounded and uniformly continuous. By $3.8, \bar{f}$ is uniformly continuous on $w \mu X$.

The next result is also based on a similar theorem in [4]. Let us 
quote a lemma [4, proposition 2.3]: every uniform space which is not precompact has an infinite uniformly discrete subspace.

3.10. For a metric space $\mu X, \mu$ can be finer than the weak derivative of some weak uniformity $\nu$ on $X$ only if (1) the set $C$ of all nonisolated points of $X$ forms a precompact subspace of $\mu X$, and (2) for any complete subset $S$ of $X-C$, the distances of different points of $S$ are bounded away from zero. Unless $X$ has uncountably many isolated points, these conditions imply that $\mu$ is a weak uniformity and $\mu=w \mu$.

Proof. First suppose that $\mu X$ satisfies (1) and (2) and has only countably many isolated points. Then every uniform covering has a uniform refinement which consists of a finite covering of an $\varepsilon$-neighborhood of $C$ and a countable discrete covering of the rest of $X$; thus $\mu$ is a weak uniformity. Consider the completion $\pi \mu X$ of $\mu X$. If $\pi \mu$ is not the finest uniformity consistent with the topology, then there is a non-uniform open covering $\left\{U_{\alpha}\right\}$. This means that there is a sequence of points $z_{n}$ such that for each $n$, no $U_{\alpha}$ contains the sphere of radius $2^{-n}$ about $z_{n}$. Since $X$ is a dense subspace, we may choose $x_{n}$ in $X$ within distance $2^{-n}$ of $z_{\alpha}$, so that no $U_{\alpha}$ contains the $2^{1-n}$-sphere about $x_{n}$. Since $\left\{U_{\alpha}\right\}$ is an open covering, the sequence $\left(x_{n}\right)$ can have no accumulation point in $\pi \mu X$. Since $C$ is precompact, it is not possible that infinitely many $x_{n}$ are in $C$. Then we may choose a subsequence-to simplify notation, suppose it is the whole sequence-so that $\left\{x_{n}\right\}$ is an infinite subset of $X-C$, which is closed in $\pi \mu X$ and thus complete, but such that no $U_{\alpha}$ contains the $2^{1-n}$-sphere about $x_{n}$. This means that we can choose $y_{n}$ in $X$ within distance $2^{1-n}$ of $x_{n}$, so that no $U_{\alpha}$ contains the $2^{2-n}$-sphere about $y_{n}$. It is therefore impossible (as before) that infinitely many $y_{n}$ are in $C$. But now we have a complete subset of $X$ $-C$, consisting of all the $x_{n}$ and all but finitely many $y_{n}$, in which distances are not bounded from zero. The contradiction proves the untenability of the hypothesis that $\pi \mu$ is not the finest uniformity consistent with the topology of $\pi \mu X$. It follows that $w \pi \mu=\pi \mu$, and since $w$ preserves subspaces, $w \mu=\mu$.

Suppose next that $\mu$ is finer than $w \nu$ for some $\nu$, but $C$ is not precompact in the uniformity induced by $\mu$. Since $w$ preserves subspaces, it is clear that $C$ is not precompact in $\nu X$ either. Therefore $C$ has an infinite uniformly discrete (in $\nu X$ ) subspace, by the proposition 2.3 of [4] which was pointed out above. This means there are an infinite subset $\left\{x_{i}\right\}$ of $C$ and a covering $u$ in $\nu$ such that the sets $\operatorname{St}\left(x_{i}, u\right)$ are disjoint. Choose $v<^{*} u$ in $\nu$, so that the sets $S_{i}=S t\left(x_{i}, v\right)$ form a uniformly discrete collection. Choose points $z_{i}$ in $S_{i}, z_{i} \neq x_{i}$, such that for some metric $d$ inducing the uniformity $\mu, d\left(z_{i}, x_{i}\right)$ converges to zero. For 
each $i$, there is a bounded uniformly continuous real-valued function $f_{i}$ on $\nu X$ such that $f_{i}\left(x_{i}\right)=0, f_{i}\left(z_{i}\right)=1$, and on $X-S_{i}, f_{i}$ is identically 1 . Define the real-valued function $g$ on $X$ as follows: if $x$ is in some $S_{i}, g(x)$ $=f_{i}(x)$; if $x$ is in no $S_{i}, g(x)=1$. Then on every element of $v, g$ coincides with some $f_{i}$; therefore by $3.8, g$ is uniformly continuous on $w \nu X$. Supposedly $w \nu$ is coarser than the metric uniformity $\mu$; but since $\left(d z_{i}\right.$, $\left.x_{i}\right)$ converges to zero while $g\left(z_{i}\right)-g\left(x_{i}\right)=1$, this is absurd. There remains the possibility that $\mu \supset w \nu$ and $X$ contains a complete set of isolated points $S$ which contains a sequence of pairs $\left(x_{i}, y_{i}\right)$ such that $d\left(x_{i}\right.$, $y_{i}$ ) converges to zero ; but evidently the above argument can be repeated in the set consisting of all $x_{i}$ and $y_{i}$, to lead to the same contradiction.

A corollary of 3.10 is that in the sequence of uniformities $\mu, w \mu$, $w^{2} \mu, \cdots$, at most two can be metric, the first and the last.

3.11. If $f: \mu X \rightarrow m E^{n}$ is a uniformly continuous homeomorphism upon a closed set, and $\mu$ is weak, then $f: w \mu X \rightarrow a E^{n}$ is a uniform equivalence.

Proof. We have that $f: w \mu X \rightarrow a E^{n}$ is uniformly continuous. Let $\nu Y$ be the image of $X$, regarded as a subspace of $a E^{n}$. Since $f$ is a homeomorphism, every open covering of $X$ is the inverse image of an open covering of $Y$; since $Y$ is closed in $E^{n}$, every open covering of $Y$ is in $\nu$. Therefore every open covering of $X$ (a fortiori, every uniform covering of $w \mu X)$ is realized by $f: w \mu X \rightarrow \nu Y$. Thus $f$ is a uniform equivalence.

3.12. Theorem. A uniform space $\mu X$ is uniformly equivalent to a [closed] subspace of $a E^{n}$, for some $n$, if and only if (1) $\mu$ is a weak uniformity, (2) $\mu=w \mu$, and ( 3 ) $\mu X$ has a uniform covering whose elements are precompact [compact] metric speces which have finite-dimensional completions of bounded dimension.

Proof. Since $w$ preserves subspaces, the necessity of the conditions is evident. Since $w$ commutes with completion, it suffices to prove the sufficiency in case $\mu X$ is complete; and by 3.11 , it suffices to construct a uniformly continuous homeomorphism upon a closed set. We may replace the covering given by (3) with a Euclidean refinement $u=\left\{U_{i}\right\}$, realized by a uniformly continuous mapping $f: \mu X \rightarrow m E^{n}$, and partitioned into $p$ discrete subcollections $u^{r}$. Each $U_{i}$ is a compact $k$-dimensional metric space and hence is homeomorphic to a bounded subset of $E^{q}, q=2 k+1$. It remains to build a mapping into $(n+p q)-$ space, as in 3.3 .

Let $e_{i}$ be $a$ homeomorphism of $U_{i}$ into $E^{q}, g_{i}$ a bounded uniformly 
continuous extension of $e_{i}$ over $\mu X$. Let $d_{i}$ be a uniformly continuous function on $\mu X$ with values in $[0,1]$, vanishing outside the star of $U_{i}$ and identically 1 on $U_{i}$. For each $x$, let the first $n$ coordinates of $h(x)$ be those of $f(x)$; let the $(n+q(r-1)+1)$ th through $(n+q r)$ th coordinates form the vector $\sum\left[d_{i}(x) g_{i}(x) \mid U_{i} \in u^{r}\right]$. On each $U_{i}, h$ is a finite sum and thus is uniformly continuous; therefore by $3.8, h$ is uniformly continuous on $\mu X$. On each $U_{i}$, hence on $X, h$ is a homeomorphism. Finally, since $f$ realizes $u$, the sets $h\left(U_{i}\right)$ form a locally finite collection of compact sets, and therefore their union is closed. By 3.11, the proof is complete.

\section{REFERENCES}

1. V. Boltyanski, On a property of two-dimensional compacta, Dokl. Akab. Nauk 75 (1950), 605-608.

2. N. Bourbaki, Topologie Générale, Act. Sci. Ind. 858, Paris, 1940.

3. - Topologie Générale, Act. Sci. Ind. 1045, Paris, 1948.

4. S. Ginsburg and J. Isbell, Some operators on uniform spaces, to appear.

5. J. Isbell, Algebras of uniformly continuous functions, to appear.

6. M. Katetov, On real-valued functions in topological spaces, Fund. Math. 38 (1951), 85-91.

7. K. Morita, On the simple extension of a space with respect to a uniformity, I, Proc. Japan Acad. 27 (1951), 65-72.

8. G. Mostow, Equivariant embeddings in euclidean space, Ann. Math. 65 (1957), 432446.

9. Yu. Smirnov, Geometry of infinite uniform complexes and $\delta$-dimension of proximity spaces, Mat. Sb. 40 (82), (1956), 137-156.

10. J. Tukey, Convergence and uniformity in topology. Ann. Math. Study 2, Princeton, 1940.

UNIVERSITY OF WASHINGTON 


\section{PACIFIC JOURNAL OF MATHEMATICS}

\section{EDITORS}

David Gilbarg

Stanford University

Stanford, California

R. A. Beaumont

University of Washington

Seattle 5 , Washington
A. L. Whiteman

University of Southern California Los Angeles 7, California

E. G. Straus

University of California

Los Angeles 24, California

\section{ASSOCIATE EDITORS}
E. F. BECKENBACH
C. E. BURGESS
M. HALL
E. HEWITT
A. HORN
V. GANAPATHY IYER
R. D. JAMES
M. S. KNEBELMAN

L. NACHBIN

I. NIVEN

T. G. OSTROM

H. L. ROYDEN
M. M. SCHIFFEI

G. SZEKERES

F. WOLF

K. YOSIDA

\section{SUPPORTING INSTITUTIONS}

UNIVERSITY OF BRITISH COLUMBIA

CALIFORNIA INSTITUTE OF TECHNOLOGY

UNIVERSITY OF CALIFORNIA

MONTANA STATE UNIVERSITY

UNIVERSITY OF NEVADA

OREGON STATE COLLEGE

UNIVERSITY OF OREGON

OSAKA UNIVERSITY

UNIVERSITY OF SOUTHERN CALIFORNIA
STANFORD UNIVERSITY

UNIVERSITY OF TOKYO

UNIVERSITY OF UTAH

WASHINGTON STATE COLLEGE

UNIVERSITY OF WASHINGTON

AMERICAN MATHEMATICAL SOCIETY

CALIFORNIA RESEARCH CORPORATION

HUGHES AIRCRAFT COMPANY

THE RAMO-WOOLDRIDGE CORPORATION

Printed in Japan by Kokusai Bunken Insatsusha

(International Academic Printing Co., Ltd.), Tokyo, Japan 


\section{Pacific Journal of Mathematics}

\section{Vol. 8, No. 1 \\ March, 1958}

Shimshon A. Amitsur, Commutative linear differential operators ......... 1

Masahiko Atsuji, Uniform continuity of continuous functions of metric

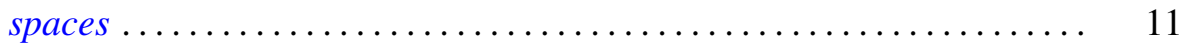

S. P. Avann, A numerical condition for modularity of a lattice . . . . . . . . . 17

Raymond G. D. Ayoub, A mean value theorem for quadratic fields........ 23

Errett Albert Bishop, Subalgebras of functions on a Riemann surface ..... . 29

Shaul Foguel, The relations between a spectral operator and its scalar

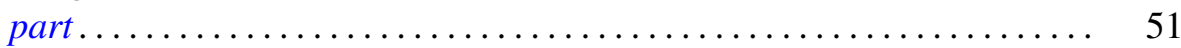

John Rolfe Isbell, Euclidean and weak uniformities ................. 67

Samuel Karlin and James L. McGregor, Many server queueing processes with Poisson input and exponential service times .............. 87

Paul Joseph Kelly and Ernst Gabor Straus, Curvature in Hilbert geometries....................................... 119

John W. Lamperti, Stationary measures for certain stochastic processes . . . 127

Richard Scott Pierce, Distributivity and the normal completion of Boolean

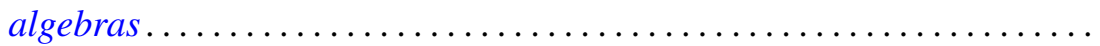

F. M. Ragab, Transcendental addition theorems for the hypergeometric

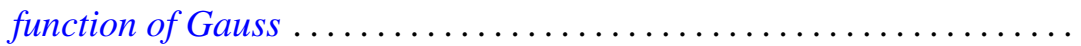

William T. Reid, Principal solutions of non-oscillatory self-adjoint linear differential systems ................................ 147

Maurice Sion, On general minimax theorems .................... 171

Chien Wenjen, On semi-normed ${ }^{*}$-algebras .................... 177 